\title{
Baroque
}

BAROQUE 09-10|1980

Méthodologie

\section{Baroque de surcharge et baroque de mouvement}

\author{
Jacques Vanuxem
}

\section{(2) OpenEdition}

Journals

Édition électronique

URL : http://journals.openedition.org/baroque/533

DOI : 10.4000/baroque.533

ISSN : 2261-639X

\section{Éditeur :}

Centre de recherches historiques - EHESS, Éditions Cocagne

\section{Édition imprimée}

Date de publication : 1 mai 1980

ISSN : 0067-4222

Référence électronique

Jacques Vanuxem, «Baroque de surcharge et baroque de mouvement », Baroque [En ligne], 09-10 |

1980, mis en ligne le 15 mai 2013, consulté le 10 décembre 2020. URL : http://

journals.openedition.org/baroque/533; DOI : https://doi.org/10.4000/baroque.533

Ce document a été généré automatiquement le 10 décembre 2020.

(c) Tous droits réservés 


\title{
Baroque de surcharge et baroque de mouvement
}

\author{
Jacques Vanuxem
}

\section{NOTE DE L'AUTEUR}

Je remercie ici M. J-P. Martinon qui m'aida dans la mise au point de cet article. M. P. Gil assura la retranscription de mon exposé du colloque de Montauban.

\section{Communication}

\section{La passion du baroque}

1 Je n'ai pas participé depuis 1966 aux sessions organisées par M. Castan ; j'avais déjà, dans la première session, donné quelques témoignages sur Le Bernin et son art apprécié par la France, et en particulier sur le témoignage de l'abbé de La Chambre. J'avais insisté sur le Baroque de mouvement. À la session suivante, en 1966, je vous avais entretenu du théâtre et des fêtes, des décors et là j'avais déjà fait état de la notion des deux Baroques qui me parait évidente : Baroque de surcharge et Baroque de mouvement. Mais avant de revenir sur ces deux notions, je voudrais évoquer la mémoire de celui qui a présidé ma première communication, de 1963. M. Victor-Lucien Tapié, inoubliable auteur de «Baroque et Classicisme».

2 Pierre Charpentrat date de 1967 l'année où parut la première édition de l'ouvrage de V.L. Tapié, la marche triomphale du Baroque, le me permets de proposer une date un peu plus ancienne qui ne modifie rien à l'immense valeur du travail de M. Tapié.

3 Au contraire de M. Charpentrat, que je n'ai jamais rencontré mais avec qui j'ai correspondu, je connaissais bien M. Tapié et j'avais pour lui la plus déférente amitié. Il m'honora de l'une de ses dernières sorties. 
4 Nous n'avions cependant pas toujours été d'accord sur la notion même de Baroque. M. Tapié parlait de style Baroque. Pour moi, le Baroque n'est pas un style comme l'est le style Roman, le style Gothique, c'est une tendance très générale et nous y reviendrons. D'autre part, j'ai parfois discuté la géographie de $\mathrm{M}$. Tapié pour qui les ports et les villes sont moins baroques que les régions montagneuses ou les campagnes.

5 Je vous avoue tout de suite avoir l'esprit le moins philosophe du monde : je suis beaucoup plus un visuel qu'un intellectuel et pour moi le Baroque est avant tout un choc et un éblouissement: un choc que l'on éprouve en visitant un édifice postérieur à la Renaissance et, antérieur au retour à l'Antique. L'admiration l'emporte, admiration tout à fait irraisonnée d'abord; que ce soit devant l'église de Vicoforte ${ }^{1}$ ou devant l'escalier de Brühl ; l'admiration est d'abord irraisonnée, puis l'on cherche à démêler les ornements et le mouvement engendré par les formes, par les contrastes des couleurs.

Je diviserai cet exposé en deux parties :

6 Dans la première, j'expliquerai rapidement ce qu'a été pour moi le Baroque. La seconde partie sera consacrée au texte qui vous intéresse, que j'ai écrit pour l'Encyclopédie de la Pléiade et où j'ai cherché à analyser les deux aspects du Baroque: la surcharge et le mouvement.

7 Né à Lille, dans une maison familiale riche en œuvres d'art baroques, ayant vécu dans le Pas-de-Calais, connaissant dès mon âge le plus tendre l'abbaye de Valloires, œuvre pour la décoration d'un artiste allemand, tout me familiarisait dès ma jeunesse avec le Baroque. À propos de Valloires, je ferai remarquer qu'il vient de paraitre dans la Revue des Monuments Historiques, un article assez confus sur l'abbaye de Valloires que l'auteur situe en Thiérache. Or, cette abbaye se trouve dans le Ponthieu, éloigné d'une centaine de kilomètres de la Thiérache².

Dans un voyage que je fis à Rome en 1925, je fus beaucoup plus ébloui par les monuments baroques de la Ville Éternelle que par les restes de l'Antiquité ; on m'avait pourtant appris à admirer les uns et à mépriser les autres.

9 Focillon fut mon maître à la Sorbonne; il avait peu l'occasion de parler du Baroque; il employait ce terme avec une extrême prudence. Avec ses étudiants je visitais, en 1928, l'église des Quatorze Saints en Franconie; ce fut pour moi, comme pour tant d'autres visiteurs, le chemin de Damas. Passionné désormais par le Baroque allemand, de 1928 à 1936, je me rendais chaque année en Allemagne. À partir de 1936, la situation politique rendait impossible tout voyage outre-Rhin. Je me consolais en cherchant à mieux connaître ce qu'il pouvait y avoir de baroque en France et je m'aperçus rapidement que ce pays serait aussi baroque que l'Italie et l'Espagne si les destructions révolutionnaires n'avaient procédé à un nettoyage par le vide véritablement insensé. Il reste encore çà et là quelques autels, quelques chaires, quelques retables, mais à Paris tout a été impitoyablement enlevé ; les églises vidées, les œuvres d'art vendues par celui qui aurait dû les protéger, Alexandre Lenoir. Les retables et les baldaquins détruits. Les Invalides, Saint-Roch, pour ne prendre que deux exemples, ne font plus aujourd'hui que la moitié de l'effet qu'ils faisaient avant 1789.

10 Je revins en Allemagne pendant la guerre et à la suite de la guerre j'y fus appelé par M. Michel François qui s'occupait alors des Beaux-Arts auprès du général Kœnig. Il m'affecta à la région de Tubingen et cette époque a été décrite de façon inoubliable par Michel Tournier dans « Le Vent Paraclet » paru en 1977 : 
11 «Notre table était présidée par un aîné dont nous aimions l'immense culture. Il connaissait mieux que personne le Baroque allemand et autrichien et aussi la douceur, l'onction cléricale, la rondeur épiscopale qui s'accordaient à un embonpoint de jouisseur spirituel et raffiné ". Jacques Vanuxem, industriel dans le civil, connut certainement, au cours de cette brève période de sa vie, l'aventure la plus exaltante que le destin pouvait réserver à cet amoureux des choses prestigieuses et vénérables. »

12 Je fis visiter à M. François ces belles églises baroques que je connaissais. Après les avoir vues, M. François me dit :

Il est inadmissible que les Français connaissent si mal de pareils chefs-d'œuvre ; il faut tenter un grand coup; je vais inviter dans notre zone la Société Française d'Archéologie, nous lui présenterons ces édifices.

13 Il en fut fait ainsi et ce congrès, qui eut lieu en 1947, fut un succès. Les congressistes furent éblouis et ressentirent le grand choc baroque.

14 Peu de jours après la fin de ce congrès, dans un hebdomadaire parisien du 11 juillet 1947, J. Vallery-Radot, décédé aujourd'hui, qui fut conservateur du Cabinet des Estampes et qui publia plus tard un recueil précieux sur les églises des Jésuites, fit un compte rendu de ce colloque :

15 «Il était réservé à M. Jacques Vanuxem de présenter aux congressistes les églises de la première moitié du XVIII ${ }^{\mathrm{e}}$ siècle, et ce n'est pas trop de dire qu'il s'acquitta de cette tâche avec la science d'un historien et la sensibilité d'un artiste.

16 Historien, sans doute, il fallait l'être pour disserter de l'école de Vorarlberg d'où sortirent les Frans Beer, les Thumb, les Mosbrugger, architectes respectivement responsables des églises de Weissenau, de Bimau et de Weingarten, ou pour évoquer Wessobrün en Bavière, la ville des grands stucateurs, les Franz Schmuzer, les Feichtmayer, les Zimmermann.

Mais, pour faire comprendre une esthétique aussi différente de la nôtre, l'historien dut céder le pas à l'artiste. C'est en effet en artiste ou en poète accessible aux nuances les plus fines et les plus délicates que $\mathrm{M}$. Vanuxem commenta le résultat de cette intime et étonnante collaboration entre architectes, peintres et stucateurs, au cours de laquelle, comme aux accents de la lyre d'Amphion, les pierres s'animaient, les façades ondulaient, les frontons se brisaient, tandis que le stuc malléable et docile se pliait aux rêves les plus audacieux d'ornemanistes à l'imagination inépuisable, et que la peinture crevait les coupoles en des perspectives plafonnantes, superposant en d'étonnant trompe-l'œil les colonnades feintes aux supports de pierre des édifices. Mais ce n'est pas tout. Il fallait dire encore, et M. Vanuxem ne l'oublia pas, les colonnes et les chapiteaux dorés, l'allègre symphonie des couleurs brillantes et claires, les balustrades qui ondulent au gré du renflement des tribunes, les vastes thèmes mystiques des compositions peintes, les chaires, croulant sous le poids des allégories, les miroirs aux bordures d'or incrustés dans les stucs, les grandes châsses de verre où des squelettes allongent sur des coussins de brocart leurs frêles ossements vêtus d'une parure d'apparat, et bien d'autres choses encore, pour montrer tout le dynamisme de cet art en mouvement que caractérise si pleinement l'absolue primauté du décor sur l'architecture. Devant les étonnantes églises de Birnau et de Zwiefalten, la première achevée en 1750, la seconde dédiée en 1755 , plus d'un songeait qu'environ à la même époque, en 1752, à Paris, Louis XV instituait le concours où devait triompher Jacques-Ange Gabriel et que de ce concours sortirait le garde-meuble aux lignes si calmes et si nobles, au décor si mesuré.

17 Tout est dit par Vallery-Radot qui distingue le mouvement et la surcharge. C'est par cet article de 1947 que Charpentrat aurait dû marquer le début du « triomphe du Baroque ». 
Dans son livre Le Mirage Baroque, Charpentrat fait plusieurs allusions à ce congrès de Souabe :

Jacques Vanuxem commençait à extraire de leur pénombre folklorique la Bavière et la Souabe, terres ignorées, dédaignées entre toutes dans la très précieuse publication de la $105^{\mathrm{e}}$ session du Congrès archéologique de France, à la rédaction de laquelle il a pris une part prépondérante.

Et plus loin il parle du rôle du Haut Commissariat à Baden, page 41. Il relève longuement que les Français occupant l'Allemagne contribuèrent à la liquidation, je le cite :

[...] d'un bien sot mythe, celui de l'Allemagne française du XvIII siècle obsédée par Versailles et uniformément classicisante. Il nous reste sur cette action parfois un peu ambiguë mais intelligente et finalement positive, un précieux document : Les actes du $\mathrm{Cv}^{\mathrm{e}}$ Congrès archéologique de France. Invitée en 1947 en Bade et en Wurtemberg, la vénérable Société Française d'Archéologie étudia non seulement les vieux sites médiévaux, Hirsau, Reichenau ou Belenhausen, non seulement les œuvres néo-classiques du Français Michel d'Ixnard Saint Blaise, Buchau, mais quelques-unes des plus riches églises baroques de Souabe, Weingarten, Bimau, Zwiefalten, Steinhausen. Le contraste est instructif entre tel discours de la séance, de clôture où un archéologue parisien s'accrocha désespérément aux traces d'influence française et les notices détaillées de Jacques Vanuxem sur les spécimens d'une architecture que la France a fort peu marquée, les premières qui aient été jamais rédigées en français ».

20 Par la suite, Charpentrat fait état de la conversion au Baroque de savants tels que Pierre du Colombier qui était spécialisé dans l'étude d'un art allemand imitant l'art français.

21 J'ai eu moins de succès avec un autre érudit français bien connu, Louis Réau, qui a beaucoup écrit sur l'art allemand mais qui a semblé irréductible au Baroque.

Pour des raisons personnelles, je dus rentrer en France, mais j'eus du mal à m'éloigner de l'art baroque allemand.

Charpentrat fait remarquer (p. 135 de son ouvrage) que je m'étais intéressé à la fantaisie des ornemanistes allemands qui ont renouvelé, grâce aux éditeurs d'Augsbourg, les motifs à la Berain.

De retour à Paris, j'entrepris de collectionner tout ce que je pouvais trouver et qui venait d'Augsbourg, gravures, livres, papiers découpés et perspectives, et d'étudier leurs rapports, avec l'architecture baroque. Le succès dépassa mon attente ; je constatais que les recueils des fêtes de Louis XIV servaient, très transposées, de thèmes aux graveurs d'Augsbourg. On édita trois fois dans cette ville le recueil des tapisseries du Roi et les devises qui figurent dans les bordures de ces tapisseries.

25 J'ai eu l'occasion de revenir bien souvent sur ce thème, mais loin d'imaginer comme Réau et du Colombier que l'art baroque allemand pouvait n'être qu'une imitation de l'art français, je montrais que les Français avaient fourni les thèmes que l'art allemand avait complètement transposés et transformés.

Il en fut de même pour les rapports avec Venise; Venise, Augsbourg et Paris furent les trois sommets d'un triangle entre lesquels les formes s'échangeaient avec surenchère de beauté.

Depuis, je fis la connaissance du Piémont où je retrouvais, surtout dans la région de Mondovi, un admirable Baroque, dont l'église de Vicoforte est le plus bel exemple.

Je fus amené à m'occuper encore du Baroque dans «l'Homme et l'Art » de la collection dirigée par M. René Huyghe ; dans la hâte de la rédaction je laissais passer une erreur qui 
me fut amèrement reprochée, ce qui ne m'empêcha pas d'être sollicité peu de temps après par des dirigeants de l'Encyclopédie de la Pléiade qui me demandèrent de rédiger un chapitre sur l'art du XVII ${ }^{\mathrm{e}}$ et du XVIII ${ }^{\mathrm{e}}$ siècle.

Je renonçais à la notion de classicisme et je me contentais de mettre tout l'art de cette époque sous la seule dénomination «l'Art Baroque». Sur ce point, je me sépare de Charpentrat qui refuse toute formule tendant à réduire le classicisme à une modalité de ce qu'on nomme le Baroque. Quinze ans après, j'ai gardé cette idée.

\section{Baroque, nature et ornement}

L'Art baroque, ainsi mis en tête de développements qui traitent de l'art occidental de la fin du $\mathrm{XVI}^{\mathrm{e}}$ siècle jusqu'au retour à l'antique, laisserait croire que je me suis rallié aux théories qui mettent l'étiquette baroque sur toutes les créations artistiques de ce temps, et que je me suis rallié aussi aux théories qui, opposant l'art classique au baroque, admettent, pendant une assez longue période, la victoire de celui-ci sur celui-là. Il n'en est rien; pour moi, classique et baroque ne marquent pas des conceptions opposées par définition, mais seulement des tendances très générales. J'adopte la seule étiquette baroque parce que l'art $\mathrm{du} \mathrm{xvII}^{\mathrm{e}}$ siècle et de la première moitié du siècle suivant s'est dirigé surtout vers ce que nous pouvons appeler baroque, mais je ne veux rien affirmer davantage.

Il ressort cependant des textes du temps, un sens limité et précis qui convient bien à certaines tendances des arts : irrégularité, singularité, effort pour impressionner, sans souci d'être raisonnable. Ainsi resserrée, la notion de baroque est parfaitement précise et utilisable.

La Renaissance avait été dominée par le désir de retrouver les formes de l'antiquité ; on ne pouvait, au $\mathrm{XVI}^{\mathrm{e}}$ siècle, trop admirer les monuments de la Rome antique. Certains artistes avaient su retrouver l'esprit même de l'antiquité et la prolongeaient.

Cependant, l'admiration de l'antiquité avait entraîné les artistes dans d'autres voies, celles ouvertes par Michel-Ange et le maniérisme. Ce style admirable étirant et faisant mouvoir les formes humaines dans des décors irréels inspira par réaction le désir de revenir à la nature ; ce retour au naturel, en passant par-dessus les modèles antiques, fut le seul but avoué du Caravage comme du Bernin; les documents contemporains abondent pour l'affirmer. Même en architecture, le Bernin se flattait d'avoir pour principal modèle le corps humain et ses proportions. L'église de Vicoforte, pour prendre un exemple de forme elliptique, représente la femme avec les bras allongés de long de son corps.

L'homme dans son naturel, dans sa variété et son mouvement, peut offrir bien des aspects étranges et s'il est le principal modèle de l'art, il n'est pas le seul; la nature animale, végétale et minérale offre toutes sortes, de bizarreries, d'irrégularités. Dégagé des modèles antiques - qui d'ailleurs ne les avaient pas négligés - l'artiste peut les contempler et s'en inspirer. Au cours du XVII et du XVIII siècle partout on collectionna et on étudia les raretés et ce qui est inhabituel. Les savantes recherches de M. Baltrusaitis sur les « Aberrations » de ce temps, apportent les plus suggestives précisions à ce propos. Les pays qui n'auraient été que raisonnables et équilibrés sont ceux où le goût du baroque et de l'inattendu a été le plus répandu: la Hollande dont les ports étaient pleins d'étranges merveilles, la France où le P. Bouhours, dans son premier entretien d'Ariste et 
d'Eugène paru en 1671, vantant les tempêtes de la mer, admire les coquillages qu'elle laisse sur le sable :

Ces coquillages qui parent si bien le fond de la mer - dit le P. Bouhours - et où l'on voit une variété infinie de figures et de couleurs, sont des productions de la nature, fort jolies et fort bizarres; ce sont des ouvrages de l'art tant elles sont régulièrement travaillées.

Quelques autres textes du même temps montrent combien partout on recherchait l'étrange et le bizarre dans la France dite classique. En 1665, un amateur particulièrement éclairé, Charles Patin, est amené dans son « Histoire des Médailles » (p. 49) à citer les plus belles productions de la nature et de l'art: les pierres précieuses et les curieuses, les oiseaux et les poissons desséchés, les coquilles, les porcelaines de la Chine, les ouvrages du Japon.

Dans le Mercure Galant de 1679, année de la paix de Nimègue et de la plus grande gloire de Louis XIV, on lit des considérations tout à fait curieuses sur la peinture qui, comme toute forme d'art, a son origine dans la nature. L'auteur fait remarquer que la nature ellemême fait des images : «les racines de l'olivier, du fresne, du noyer étant travaillées et polies, représentent mille figures bizarres qui sont l'ouvrage de la nature... » et il rappelle les collections qui réunissaient de pareilles raretés.

Un auteur dramatique, Palaprat, a écrit en 1712 l'essentiel sur ce sujet : «Le goût du temps en toutes choses ne me parait pas ennemi des irrégularités : il suffirait de la fureur qu'on a pour les ouvrages de la Chine pour prouver que les imaginations les plus bizarres plaisent plus que les desseins suivis et corrects...

Les arts ne font qu'imiter la nature... Il semble qu'il y ait un «je ne sais quoi » de plus piquant dans les caprices de la nature que dans ses opérations exactes; ce doit être un favorable préjugé pour les, ouvrages de l'esprit en un siècle où les choses nouvelles, extraordinaires et bizarres ont pris un si grand empire.

De tous ces témoignages, il ressort que des modèles nouveaux extraordinaires, comparables aux objets exotiques, viennent de la nature même; le contact avec les formes naturelles conduisait au bizarre ou au surprenant. Ce goût apparaissait dans tout l'Occident: Palaprat rappelait combien les choses de la Chine étaient à la mode en France. Elles l'étaient encore davantage en Hollande ou en Angleterre qui avaient de grandes flottes commerciales qui allaient jusqu'en Extrême-Orient. Dans un texte écrit en 1685, l'Anglais Temple explique comment les Chinois voyaient la beauté de leurs jardins et se moquaient de la symétrie Qu'on observait « fort exactement » en Europe

[...] ils occupent tout leur esprit, qu'ils ont extrêmement inventif, à imaginer des figures. Qui soient d'une grande beauté et qui surprennent la vue, mais dans lesquelles on ne peut remarquer cet ordre et cet arrangement à quoi nous regardons tout aussitôt.

Temple reconnaissait ainsi que l'irrégularité et le dissymétrique pouvaient être d'une grande beauté.

Ce souci de la nature qui a mené au bizarre, mènera à la surabondance et à la surcharge ; l'artiste, dégagé des entraves que lui imposait l'imitation de l'antique, laisse aller ses forces créatrices. Il utilisera toutes les richesses dont il peut disposer : matériaux divers, couleurs, le tout dans une abondance foisonnante... La surcharge et l'ornement susciteront de nombreuses polémiques, car on allait ainsi à l'encontre du goût pour l'architecture soi-disant dépouillée issue de l'antique; mais précisément nous trouvons un terrain fort solide pour appliquer l'étiquette baroque. Nous aurons donc incontestablement un baroque de surcharge qui prolonge le goût pour le bizarre et s'y 
allie. C'est aussi un retour vers la nature, et dans cette surcharge décorative, on retrouve souvent des formes inspirées plus ou moins directement de la végétation ou des animaux, en particulier dans les encadrements et les cartouches.

Le goût pour le bizarre était parfois contrarié par le désir de se conformer aux fameuses règles inspirées des ouvrages anciens, par le désir de montrer des édifices harmonieux, mais la fantaisie avait toujours sa p1ace dans l'ornement. Fort curieuses sont les pages que le Père Binet, jésuite, a consacrées à l'architecture dans son «Essai des merveilles de la nature » dès 1621. Après avoir vanté les mérites de la bonne architecture qui doit respecter l'eurythmie et 1a bienséance, après avoir rappelé les principes des « ordres » il précise que dans les édifices on doit laisser la place aux peintres, aux décorateurs et ail ajoute que

[...] quand la verve saisit leur pinceau, ils font des harpyes dont les queues aboutissent en floccards à costes, revestues de feuilles crêpelées, de volutes garnis de rosaces, de candélabres d'où sortent des rinceaux de feuillages délicats et fort esgayés, qui porteront des petits enfants assis bien enjoués et follastrant ensemble ; des bouillons de fleurs sortant de feuillages et de là certaines moitiés d'animaux inconnus, demy hommes finissant en bestes brutes, mille caprices, qui sont mieux reçus que les vérités mêmes, car il semble qu'on se délecte à être trompés.

Ces lignes du Père Binet indiquent ce qui sera une pratique générale dans l'art du XVII et du XVIII ${ }^{\mathrm{e}}$ siècle, même lorsque l'architecture vise à reproduire les " ordonnances » issues de l'antiquité, l'artiste décorateur aura toujours toute liberté pour ses « caprices » et pour ses fantaisies. La France de Louis XIV le prouve; si elle a visé à reproduire la majesté romaine dans ses édifices, elle n'a pas entravé la liberté des ornemanistes. Ceux-ci en ont usé et ont donné des exemples pour toute l'Europe.

41 En 1671, un critique exprimera la même idée que le P. Binet. Dans sa « Guerre des Auteurs anciens et modernes », Gabriel Gueret parlera (p. 203) « des irrégularités affectées... des caprices de ces grands Peintres qui égayent leur imagination sur des grotesques après l'avoir fatiguée sur les grands desseins ».

De nos jours, Focillon dans sa «Vie des Formes » a repris ces idées et a montré le lien qui existe entre les représentations étranges issues d'organismes vivants et la surcharge de l'ornement...

Elles vivent par elles-mêmes, avec intensité, elles se répandent sans frein, elles prolifèrent comme un monstre végétal... Elles se détachent en s'accroissant, elles tendent de toutes parts à envahir l'espace... Ces caractères sont remarquables et même saisissants dans l'art ornemental.

Un baroque ainsi compris contient en lui-même une autre tendance qui, comme la surcharge, rejoindra le bizarre et l'inattendu, celle qui fut inaugurée dans l'architecture par le Borromini et Le Bernin, et vise à suggérer le mouvement. Il n'est possible, le plus souvent, que de suggérer car les formes restent fixes mais, pour les décors de théâtre, la suggestion peut être dépassée. Le Bernin fut l'auteur de décors où l'on voyait d'étonnants changements sous les yeux du spectateur. Dans les fontaines des places publiques et des jardins, l'eau coule et parfois anime des automates. Nous rejoignons ici les recherches de M. Jean Rousset. En étudiant le Baroque littéraire, M. Rousset s'est fréquemment référé à l'histoire des arts. Étayant ainsi largement son étude du Baroque du mouvement et de la métamorphose, il a pu multiplier les rapprochements suggestifs entre ce Baroque et l'étrange, l'inattendu. 
44 Nous croyons donc que la quête du Baroque amène à retrouver ce que dans leurs créations, les artistes du XVII ${ }^{\mathrm{e}}$ siècle ont pu faire en dépassant l'imitation de l'antique : se laissant guider par des formes naturelles vers l'inattendu et le bizarre, ils ont recherché la surcharge ou suggéré le mouvement.

45 Le Baroque est une tendance purement formelle, fortement soumise à la personnalité de l'artiste. $\mathrm{Au} \mathrm{XVII}{ }^{\mathrm{e}}$ siècle un artiste est baroque quand il veut, se calmant quand il le faut, en se ménageant toutes les nuances intermédiaires. Si nous regardons des œuvres éphémères où nulles contraintes ne régnaient, nous verrons avec quelle aisance le même artiste passait de ce que nous appelons le classique au Baroque, son tempérament naturel.

Cette pratique était si générale que pour ces décors de fêtes, un grand théoricien jésuite, Menestrier, l'érigeait en principe.

Pour élever des machines qui ne servent qu'aux décorations, on n'est pas obligé de suivre les règles exactes de l'architecture... On peut imiter les anciennes grotesques...»

La liberté donc pour les édifices provisoires, pour la décoration. Les mêmes artistes devaient être capables de jouir de cette liberté ou au contraire de se plier aux règles de l'antique.

47 Comme on le voit, il est bien difficile dans ces conditions de parler de «style » baroque puisqu'il ne s'agit que d'une inflexion, d'une tendance. À l'intérieur de cette tendance très générale, on peut définir quelques styles, tels celui du Borromini, celui de Churriguera et plus tard la rocaille oui n'est pour moi, même sous son nom allemand de Rococo, qu'un style d'ornement décoratif.

48 L'étiquette "art baroque» nous convient parce que souvent celui-ci représente la tendance dominante dans l'art de ce temps, soit par la surcharge, soit par le mouvement, soit par le goût pour ce qui est surprenant dans la nature. Le baroque a fleuri dans des époques de troubles et d'instabilités. Il faut citer cet extrait du mandement d'un évêque, à propos d'un autel votif

[...] Nous voulons élever à la gloire de ce saint le trône qu'on s'était contenté de lui vouer. La disette qui semblait devoir nous absorber dans le soin des pauvres et mettre par conséquent obstacle à l'accomplissement de nos religieux desseins, en facilite aujourd'hui l'exécution par l'abondance des matériaux et des ouvriers qui n'ont point été employés à d'autres œuvres, et dont elle nous a laissé le choix à des prix plus modérés pour ce monument de notre piété....

Le mandement d'où ces lignes sont prises est daté du 10 avril 1710 : il a comme auteur l'évêque Pierre Sabatier et concerne l'autel Saint-Jean-Baptiste élevé sur les dessins du fameux ornemaniste Oppenord dans la cathédrale d'Amiens.

Si l'on veut saisir le vrai mode d'expression des tendances baroques au XVII et au XVIII siècle, ainsi que les styles qui s'y accrochent, il faut considérer l'extraordinaire diffusion des livres gravés et accessoirement des objets décoratifs facilement transportables, audelà des frontières et dans des pays de confessions différentes qui donnaient des idées et des modèles à tous les artistes.

Certains de ces livres gravés étaient consacrés à l'ornement. Sans échelle, ils contenaient un répertoire de formes bizarres qu'on pouvait utiliser pour tous les usages. Des livres d'emblèmes ou de devises, non moins étranges, venaient surtout des Pays-Bas. Des livres de fêtes reproduisaient de curieux édifices imaginaires ou provisoires et donnaient envie de perpétuer, dans des constructions durables, ces créations fantastiques, et c'est ce qui fut fait. L'ornement, la Fête et le décor dominent véritablement tout l'art de ce temps. 


\section{Baroque et rococo}

51 quelques objections aux idées qui viennent d'être rappelées. Il nous dit avoir ressenti le choc initial pour cet art à l'église des Quatorze Saints comme moi-même. Il croit que cette église est l'expression d'une civilisation du rococo : pour moi, je pense que le rococo est moins l'expression d'une civilisation qu'un style décoratif. Tout le siècle des Lumières ne peut pas être mis sous l'étiquette rococo. Le décor rocaille continue le goût du XVII siècle pour les bizarreries de la nature. Mondon est le premier à avoir appliqué le mot rocaille au décor naturaliste déchiqueté et dissymétrique. Il a été abondamment imité à Augsbourg puis par Lajoue et Boucher.

y pour moi une permanence et des rapports constants entre les différentes formes d'art au XVII et au XVIII ${ }^{\mathrm{e}}$ siècle. Rome a eu la primauté, mais les diverses régions d'Europe ont continué avec originalité et grandeur. C'est toute cette production que nous appellerons simplement baroque.

Exemples : Pl. IV à VII

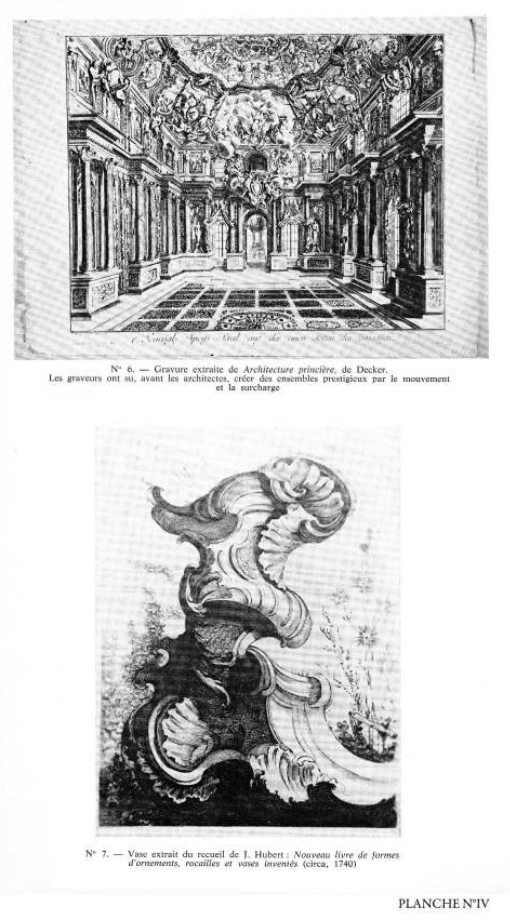




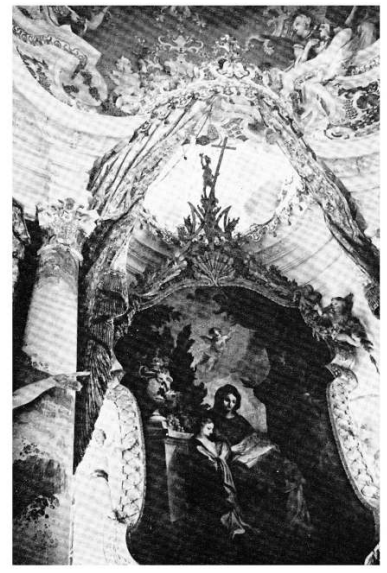

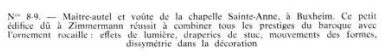

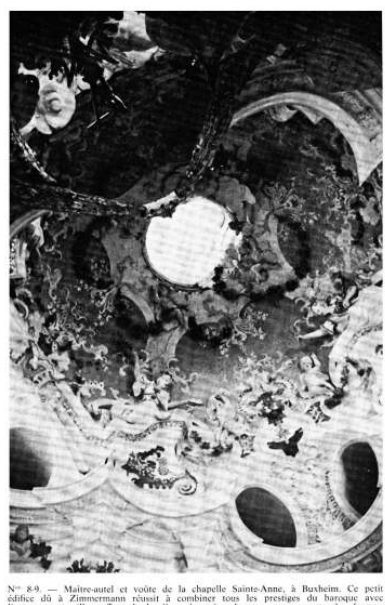

PLANCHENVI 

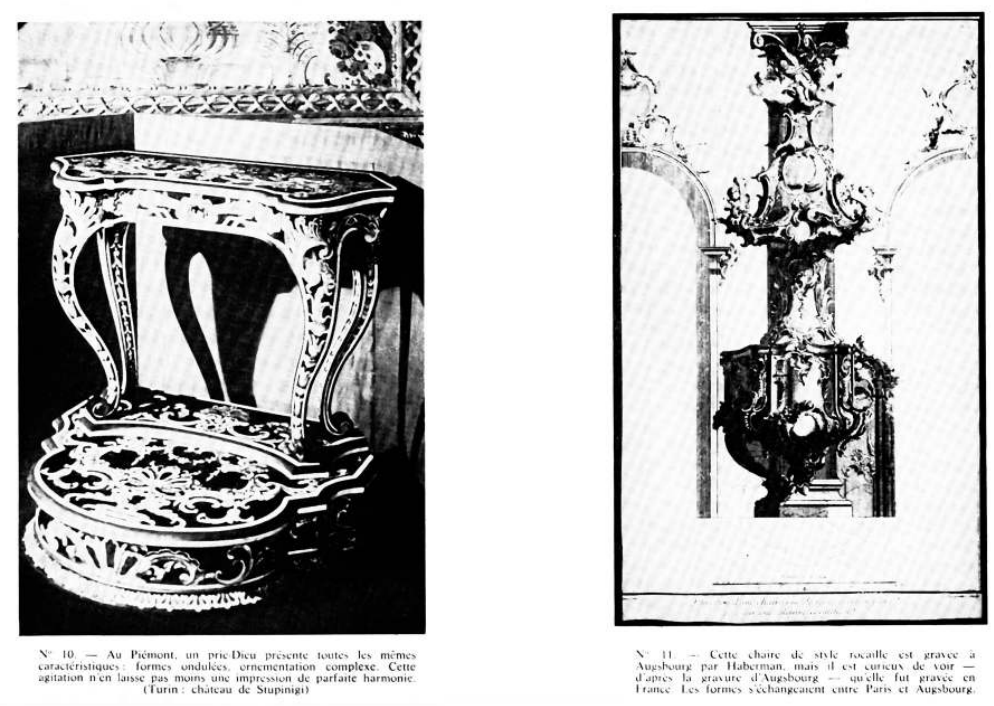

PLANCHE N"VII

\section{Discussion}

54 - CASTAN : Ici même, il y a quelques années, M. Mesnard disait « le Baroque, c'est un prolongement du grand courant né de la Renaissance ». J'aimerais que M. Vanuxem nous précise sa pensée à ce propos. Nous avons très bien vu qu'à partir de la naissance du baroque il y a un grand courant qui va, historiquement, jusqu'à la fin du rococo : ce courant est-il dans la continuité de la Renaissance?

55 - VANUXEM : Il s'agit d'un élargissement de la Renaissance. Le Baroque continue la Renaissance mais e. insistant sur la richesse de la décoration dans les palais et les églises. Mais revenons à Rome et constatons que de grands édifices commencés à l'époque de la Renaissance - Saint-Pierre et le Jésus - se sont admirablement adaptés à une décoration baroque, par contre, en France la petite échelle de la décoration Renaissance ne s'est pas toujours alliée à l'importance de l'édifice, c'est très remarquable par exemple à Blois dans la différence de traitement architectural entre l'aile François $\mathrm{I}^{\mathrm{er}}$ et l'aile de Gaston d'Orléans, il n'y a alors que juxtapositions de traitements architecturaux différents.

- MARTINON : La question de la différence de traitement et d'échelle, d'une période architecturale à une autre est fort importante mais il me semble que si nous entreprenons de nous y aventurer, nous pourrions courir le risque de préluder à l'intitulé d'un autre colloque dans lequel les architectes auraient leur mot à dire. De même si nous nous engagions dans l'analyse des résurgences, des réminiscences et des interprétations du terme « antique » dans ses relations variables et contradictoires avec le baroque, il serait tout aussi pertinent de parler du Laocon que des livres d'architecture de Vitruve !

57 - CASTAN : Pourtant, j'aimerais faire préciser à M. Vanuxem ce mot d'antique ?

58 - VANUXEM : L'antique, c'est aussi bien la sculpture agitée ou tragique de Laocon que l'architecture de Vitruve ${ }^{3}$. 
59 - CASTAN: Comment peut-on parler de Vitruve, le texte étant accessible, mais évidemment sans aucune illustration.

60 - VANUXEM : Chacun illustrait Vitruve à sa façon, mais les interprétations étaient divergentes. L'illustration rendait le texte utilisable pour un public lettré, c'est ce qu'a fait Perrault, mais aussi bien d'autres avant et après lui. Chacun construisait une image de l'antiquité, à la fois grâce à la lecture du texte et grâce à la connaissance qu'on pouvait avoir des monuments antiques.

61 - PREMIER INTERVENANT ${ }^{4}$ : Revenons au baroque par le biais de Perrault et évidemment du voyage du Bernin à Paris. Que pensez-vous du problème que pose la colonnade du Louvre?

62 - VANUXEM : La colonnade du Louvre est de Perrault et non de d'Orbay qui ne fut que le chef de chantier. Il semble que la colonnade de Perrault, puisque les colonnes étaient isolées et dégagées, faisait plus d'effet que le projet de colonnade du Bernin qui proposait des colonnes engagées.

63 - DEUXIÈME INTERVENANT : Ce détour nous fait revenir au fameux débat :

64 Baroque et classicisme et à la volonté de certains chercheurs de supprimer le concept de classicisme. Je me réfère aux historiens de la littérature française du XVII ${ }^{\mathrm{e}}$ siècle, je pense surtout à Spitzer qui, approximativement, écrit: «le classicisme français n'est qu'un Baroque dompté ». Je crois que le débat consacré au Baroque littéraire en France a été dans une large mesure un débat mal engagé parce que l'on s'est fondé, dès le départ, sur une conception $\mathrm{du} \mathrm{XVII}^{\mathrm{e}}$ siècle comme siècle du classicisme. Il s'est agi pour les partisans d'un Baroque littéraire de retrouver un petit espace, dans ce siècle identifié au classicisme et d'introduire le Baroque au début du XVII siècle : il s'agit avant tout d'une histoire de succession. Au lieu de vouloir situer le Baroque, à tout prix, par rapport à un classicisme et donc d'opérer un découpage chronologiquement situable au cours du XVII siècle, il serait plus intéressant de tirer profit de deux autres concepts celui de la coexistence entre des formes stylistiques différentes et celui d'une tendance générale qui serait baroque et qui correspondrait à une attente du public - un climat esthétique et moral si vous voulez. Mais cette tendance générale est masquée, est contraire par une volonté classique qui canaliserait, qui calmerait la furia baroque. Les attaques de Boileau contre Scudery dans l'art poétique n'auraient pas beaucoup de sens s'il n'y avait pas encore en 1674 une très grande attente dans le public, une très grande intimité avec les formes et le langage baroque.

65 - VANUXEM : Boileau et Chapelain avaient la même vision des chose, mais Chapelain n'est ni baroque ni classique, il était mauvais. Vous parliez de Boileau et je pensais aux quatre premiers vers du Chant III de l'Art poétique :

Il n'est point de serpent ni de monstres odieux,

Qui par l'art imité, ne puisse plaire aux yeux :

D'un pinceau délicat l'artifice agréable

Du plus affreux objet fait un objet aimable.

Par exemple j'ai écrit, il y a une vingtaine d'années, un article dans la Revue d'histoire littéraire ${ }^{5}$ sur le décor de l'Iphigénie de Racine lors de la fête de 1674 . J'ai voulu démontrer qu'Iphigénie avait été représenté dans un décor baroque avec des guéridons dorés, des vases, de fleurs, des girandoles et des guirlandes. Ces éléments ne s'alliaient pas à cette tragédie assez sinistre, à l'antique, puisqu'il s'agit d'un sacrifice humain. Voilà l'ambiguïté pour nous à qui l'école a appris: le $\mathrm{XVII}^{\mathrm{e}}$ siècle est classique puisque la 
tragédie classique coexiste avec ce que vous avez appelé une attente du public, avec une sensibilité à l'espace décoré qui est proprement baroque. C'est un exemple, on pourrait en trouver d'autres. Chine des Jésuites, les présents qui en furent rapportés ont été goûtés, mais il est difficile de préciser dans quelle mesure au XVII siècle ces objets furent appréciés. Ce sont des curiosités exotiques : par exemple les coquillages qui devinrent des objets d'art à la fin du $\mathrm{XVII}^{\mathrm{e}}$ siècle et surtout au XVIII ${ }^{\mathrm{e}}$ siècle. Le goût pour la Chine est une expérience du Baroque mais il faudrait alors un autre colloque pour préciser l'apport de la Chine et de ses formes d'art $a u X V I I^{e}$ et au $X^{2}{ }^{e}{ }^{e}$ siècle : il s'agit bien sûr du goût pour les formes rares et lointaines.

- AUZIAS : J'aimerais que vous précisiez votre propos au sujet du texte du Père Binet que vous avez cité au cours de votre conférence.

70 - VANUXEM : Le Père Binet dans sa description ${ }^{6}$ parle de harpies et de chimères. Ces monstres se réfèrent à des modèles et à des grotesques antiques. Je ne crois pas que le Père Binet s'intéresse aux sculptures du Moyen Age, mais ce oui me semble important dans ce texte, ce sont les phrases finales : « il semble qu'on se délecte' à être trompé ». Je me demande s'il n'y a pas, là une prise de conscience du plaisir à être trompé par l'art : la peinture est faite pour tromper agréablement l'œil et la poésie trompe agréablement l'esprit.

71 - MARTINON : Il s'agit de l'artifice et des rapports spécifiques que le Baroque entretient avec la nature et ses modes de représentation. Les constructions, les décorations baroques présentent des jeux subtils réitérant par la pierre, le stuc ou la porcelaine, la nature sauvage et ses moments exceptionnels de floraison et d'épanouissement coloré. D'autre part, vous avez évoqué les collections de curiosités de cette époque. Représenter l'exception stylisée mais aussi conserver les objets curieux me semble bien d'esprit baroque.

72 - QUATRIÈME INTERVENANT : Je voudrais ajouter que ces collections de curiosités avaient quelquefois, dans l'esprit des collectionneurs une signification plus profonde que celle du simple plaisir muséographique le pense à Rodolphe II qui collectionnait les pierres précieuses et les objets d'art non seulement pour les admirer mais surtout pour prévenir les maladies, pour se guérir ou pour se protéger de forces magiques. On a continué à l'époque baroque à considérer les phénomènes exceptionnels que produit la nature comme étant intimement liés au destin de l'homme. De là des interférences et des débordements d'ordre sympathiques et magiques entre des phénomènes naturels et leurs correspondances, leurs résonances par rapport à l'existence de l'homme baroque. L'art baroque révèle certainement cette circulation de signes, cette magie - au sens ethnologique du terme - qui provoque une interpénétration des ordres naturel et humain. Le Baroque est peut-être tout à fait opposé au concept de séparation des ordres.

73 - VANUXEM : Vous avez raison, les cabinets de curiosités ont été ce que vous dites au XVIII e et au début du XVII ${ }^{e}$ siècle. C'était important pour l'homme de conserver des objets inattendus, par exemple des lézards empaillés. Ces objets avaient une valeur pour la vie de l'homme mais, de magiques et mystérieux, ils devinrent des objets scientifiques, conservés dans des cabinets construits à cet effet. 
74 - MARTINON : Et pourtant, l'homme baroque a un tout autre rapport aux objets et aux collections d'objets que celui de l'ethnologue?

75 - VANUXEM : En effet, le Baroque est curieux, mais les relations qu'il élabore entre les objets est d'ordre magique et non d'ordre rationnel. Ma curiosité baroque n'a rien de scientifique. Mais je voudrais terminer cette discussion sur le texte de Charpentrat: lorsque celui-ci parle du classicisme comme étant un court instant sur un fond durable, je n'approuve pas du tout cette proposition et je récuse toute formule tendant à réduire le classicisme à une modalité de ce que l'on appelle le Baroque. Je ne crois pas que mes vues, pour une fois, coïncident avec celles de Charpentrat.

76 - MARTINON : Il me semble, en cette fin de discussion, que nous n'avons fait qu'effleurer le sujet de la conférence de Jacques Vanuxem qui était centrée sar le Baroque de mouvement et le Baroque de surcharge. Nous avons successivement abordé des phénomènes - certes importants mais néanmoins périphériques - comme l'ambassade au Siam, les chinoiseries, les cabinets de curiosités et leurs rapports avec le magique, la lecture que le XviI ${ }^{e}$ siècle fait de Vitruve. Loin de rencontrer le sujet de la conférence, nous avons conversé à partir de chemins de traverses en tissant toute une série de digressions.

77 Mais peut-être est-ce le propre du baroque que de nous conduire vers ces lointaines régions par un mouvement propre au Baroque lui-même - et nous revenons alors au Baroque de mouvement - qui nous a entraîné vers la surcharge d'idées et d'exemples se situant au pourtour de l'insaisissable Baroque.

78 Mais, tant dans votre conférence que dans la discussion et la présentation des diapositives, trois termes revinrent souvent dans votre propos : il s'agit du mot choc que le spectateur et le visiteur éprouvent au seuil d'une église ou d'un palais baroque - et Charpentrat utilise aussi ce terme -, il s'agit ensuite du mot tendance, enfin du terme de période puisque l'architecture, la décoration baroque se situent à l'intérieur d'une période historiquement déterminée. Vous refusez, et je suis tout fait d'accord avec vous, de présenter les temples du Sud-Est asiatique, les sculptures et les portes d'immeubles 1900 comme faisant partie intégrante du Baroque ; mais encore faut-il essayer de préciser les limites et donc l'extension de ce qu'on peut appeler Baroque ${ }^{7}$. Ainsi, j'aimerais développer ces trois termes : choc, tendance, période.

Le choc, c'est-à-dire le rapport privilégié qu'un spectateur peut avoir avec une œuvre, dans l'immédiateté du premier regard ou bien grâce à une expérience sentimentale - et le terme n'est pas déprécié pour moi - pose brutalement la question du «qui regarde quoi ?». Il s'agit d'un rapport fortement valorisé dans la littérature esthétique, dans les récits de voyageurs et cette manière d'être, spécifique, en face de l'objet ou du monument, empreinte de fascination et de séduction, est bien sûr historiquement et sociologiquement, sinon psychanalytiquement, déductible. Les œuvres baroques, par leur densité d'existence, ne produisent pas les mêmes effets selon les spectateurs : ceci est un truisme, mais il faut toujours l'avoir présent à l'esprit. Voir ou ne pas voir telle œuvre, tel monument, ne pas l'aimer ou bien, au contraire, être séduit par lui dans une fulguration du regard reconnaissant un paradis monumental et une terre promise esthétiquement, est une valorisation de ce rapport et une situation charismatique grâce à laquelle le spectateur - de la surprise à la séduction - rencontre une passion. Quatremère de Quincy, au début du XIX ${ }^{e}$ siècle (1830), s'irrite de «l'aberration de jugement, de la vaine ambition de se distinguer en se frayant des routes tortueuses dans les champs de caprice et de 
l'innovation » d'un Francesco Borromini. Saint-Yves-de-la-Sapience est ici décrit comme " un morceau unique pour la bizarrerie $»^{8}$ : ici, point de choc ni de passion, mais au contraire l'irritation d'un défenseur du grec et du romain, sinon de l'égyptien. Au contraire, Charpentrat, un siècle et demi plus tard, qualifiera Borromini «d'architecte intégral » construisant, à Saint-Yves, l'édifice de la surprise par excellence qui, « d'un seul élan, du sol à la lanterne ", montre son ossature par ses palpitations mêmes. La fortune critique n'est qu'une des facettes de la variabilité des jugements, et le sociologue, par entretiens, par questionnaires ou bien par observations directes, serait à même de dénombrer la fréquence, la qualité et l'intensité des étonnements esthétiques de nos contemporains lorsqu'ils visitent Saint-Charles-aux-Quatre-Fontaines en s'ennuyant profondément ou lorsque, saisis et séduits, étonnés - dans le sens fort que le XVII ${ }^{\mathrm{e}}$ siècle attache à ce terme - ils y consacrent leur vie et leurs discours poétiques ou savants.

Bien sûr, il serait faux de croire que cet étonnement esthétique, cette découverte subite de la passion ne s'adresse qu'au Baroque. Toute forme d'art peut en être le réceptacle ; encore faudrait-il dresser le catalogue des formes artistiques focalisant de telles pratiques de séduction, à un moment de l'histoire et pour une population socialement donnée, tout en sachant que la machinerie séductrice n'est pas une caractérisation essentielle de l'objet séduisant, mais qu'elle est une des variables sociales, psychologique, psychanalytique, du sujet séduit. Ainsi la dégustation esthétique peut elle se porter, selon notre place dans la société, vers l'admiration d'un escalier du jardin de Troja, à Prague (1679) qui, grâce aux statues scandant le dénivellement, dramatise l'espace, ou bien vers l'admiration de l'escalier de la Maison de verre construit par Pierre Chareau et Bernard Bijovet en 1928, à Paris. La variabilité du regard esthétique et des classements qui en résultent - une taxinomie esthétique, pourrions-nous dire - serait l'objet d'une sociologie du goût des diverses classes sociales. Charpentrat, dans Le Mirage Baroque, en parle un petit peu, mais d'une manière ponctuelle et non systématique. Il faudrait ajouter, pour être complet, que le problème n'est pas si simple et qu'une théorie de la variabilité des discours admiratifs devrait être prise en compte ${ }^{9}$ : une femme du monde ne manifeste pas de la même manière son admiration devant la beauté et la richesse des églises baroques que le P.Crasset, cité par Jacques Vanuxem, défendant avec véhémence cette même richesse mettant en scène une pédagogie spirituelle. Ainsi, le « choc » peut-il être à la fois une révélation esthétique à partir de la perception directe d'un édifice et le résultat d'une situation d'inculcation sociale - d'autorité scolaire ou religieuse - incitant le spectateur à l'admiration à partir d'une photographie, d'une carte postale, d'une image ou d'un discours bien tempéré.

81 La tendance pose d'autres problèmes que je ne puis aborder ici; pourtant, il s'agit d'un choix conscient, institutionnellement réglé mais aussi inconscient, qu'un artiste peut faire à l'intérieur du catalogue des formes qui sont co-présentes à son époque. L'étude du regard du créateur sur les modèles passés et contemporains de son temps est fondamentale. Encore faudrait-il prendre en compte toutes les variables utilisables par une sociographie de l'artiste : son origine sociale, les maîtres avec lesquels il travaille ou qu'il admire, ses voyages, la connaissance qu'il peut avoir du catalogue des formes, des ornements, des techniques de son art disponible à son époque. La tendance est alors en rapport avec la liberté du créateur: la détermination de ses choix, de ses modèles historiquement présents est tout aussi programmée que les admirations du spectateur en face d'une œuvre d'art. Mais l'artiste peut aussi transformer, enrichir le catalogue des formes, multiplier les variations qui ne soient pas seulement la répétition de variations 
antérieures. Le cheminement de l'invention passe souvent, sinon obligatoirement, par les défilés de la répétition des formes. Or, pour le Baroque, la dissémination de sa définition permet une extension quasi infinie de ces variations. Il faudrait faire un recensement des jugements proférés depuis deux siècles sur le baroque afin de comprendre l'extension et la variabilité des jugements et des définitions de ce concept. En effet, les dénominations les plus savantes et les plus futiles furent exprimées à partir de ce terme générateur. Les variations, bien avant la description que Charpentrat en fit dans le Mirage baroque, bien avant les rhapsodies - telles celles de Louis II de Bavière ${ }^{10}$ - constituent des strates successives que le chercheur doit qualifier. Le chercheur devrait alors recomposer, restituer l'extension du concept... et sa patience, il devrait alors être l'homme du choc sinon de l'intuition, mais aussi celui qui peut découvrir «le contenu substantiel de la plupart des choses neuves (qui) lui paraît, plutôt quelque chose de bien connu $»^{11}$. La question de la tendance, ç'est-à-dire celle de l'extension du concept de Baroque, éprouve ainsi toute méthode de recherche de ce qui est le Baroque.

Pourtant, le concept de périodisation devrait nous aider, mais la classification historique est toujours complexe, multivariée, puisqu'elle change avec l'état de la science historique : faut-il, travailler sur de langues périodes, d'un point de vue quantitatif, faut-il au contraire s'intéresser aux limitations strictes d'une période arbitrairement décidée puisque toujours, telle une théorie de concepts suiveurs, dès que l'on essaie de préciser la période dans laquelle le Baroque pourrait être contenu, apparaissent d'autres concepts à l'horizon de la recherche: ceux de transition, de décadence, de résurgence. Tout découpage «a priori » fait naître, par l'intervention chirurgicale qu'il offre, une série de rejetons conceptuels dont on ne peut déceler la paternité, et pourtant, nous dit encore Charpentrat, quelque chose change entre l'église de pèlerinage des Quatorze Saints (dont les voûtes sont achevées en 1763) et l'église de Saint-Blaise, en pleine Forêt Noire, œuvre du Nîmois Michel d'Ixnard (1764). La première est encore baroque, la seconde ne l'est plus entièrement. Ainsi, les découpages, quels qu'ils soient, doivent être traités comme des pratiques scientifiques préformant les objets dont ils parlent. Les manières de découper l'objet d'étude en larges périodes, posent évidemment des problèmes difficiles de repérages puisque, alors, il ne s'agit plus de monographies sécurisantes pour le chercheur, ayant pour sujet l'église Saint-Charles-Borromée à Vienne, ou bien la vie et l'œuvre de Kilian-Ignace Dientzenhofer.

83 La conférence de Jacques Vanuxem a eu le mérite de nous transporter au cœur de ces difficultés et de ces interrogations sur les intermittences de la subjectivité et de l'objectivité.

84 - VANUXEM : Vous avez admirablement analysé ce que j'ai dit et vous avez précisé mieux que moi-même ce que je pense du choc et de la tendance. Le problème où je me sens beaucoup moins à l'aise est celui de la périodisation car ces découpages dans le temps ressemblent souvent à un accordéon. Parfois, il faut resserrer à l'extrême ; d'autres fois, il faut déplier largement, étendre la période : celle-ci est difficile à délimiter : il s'agit d'un nœud gordien. À propos de la notion de choc, comme vous l'avez précisé, il est tout à fait indiqué de citer le texte du P. Crasset ${ }^{12}$ :

[...] du moins on ne peut nier que le peuple et les personnes grossières dont le nombre est incomparablement plus grand que celui des savants et des spirituels ne soit excité à louer et à honorer Dieu par les riches ornements de nos églises. Ces temples si spacieux, ces autels si bien parés, ces cérémonies si augustes, ces, vêtements si riches et si précieux, tous ces vases d'or et d'argent, toutes ces images 
si bien travaillées ne contribuent pas peu à conserver la majesté de nos sacrements et à imprimer du respect pour les choses saintes [...] les palais princiers, il faut créer un choc pour que le «vulgaire " soit très fortement impressionné par l'importance de l'église ou du palais royal. Je crois que le P. Crasset a bien compris ce que pouvait être le choc lorsqu'on rentre dans une église baroque.

86

CASTAN : J'avais une inquiétude à propos de « l'insaisissable baroque ». La raison d'être de ces journées est justement de dépasser l'idée banale de l'insaisissable baroque et notre défi, dans cette réunion, c'est justement de croire que ce qu'on dit insaisissable est en réalité saisissable, mais par de nouvelles voies, par exemple, par l'analyse du discours sur le Baroque. Il s'agit d'un pari épistémologique, décisif à mes yeux, afin d'entrer dans une nouvelle étape, dans une nouvelle aventure scientifique. C'est pourquoi je suis d'accord avec la deuxième partie de l'intervention de Martinon et avec la réponse de Jacques Vanuxem s'appuyant sur le texte du Père Crasset: il s'agit, comme Vanuxem le fait, d'entrer dans le Baroque comme on entre en religion, et de participer du dedans, à l'aventure historique baroque à laquelle s'identifie notre aventure scientifique d'aujourd'hui.

\section{NOTES}

1. J. VANUXEM : L'église de Vicoforte, inCongrès archéologique du Piémont,Paris,1978,pp. 470-484.

2.

a publié un important numéro consacré au « baroque en France »,

février 1979. Cette revue apporte, dans cette livraison, des précisions Douvelles, en particulier sur la décoration des églises de certaines provinces.

3. FrançoiseFICHET :La théorie architecturale à rage classique,Bruxelles, Mardaga,1979.Françoise СноАу: Alberti et Vitruve,Architectural design,vol. 49. N 5-6, 1979,pp. 26-35.

4.

Ces interlocuteurs ne peuvent être identifiésà partir des bandes magnétophoniques sur lesquelles fut enregistrée cette discussion. Nous nous en excusons auprès des intervenants.

5. JacquesVANUXEM : « Racine, les machines et les fêtes »,R.H.L.juillet-septembre 1954.

6. BINET : «Essay des merveilles deNatureetdes plus nobles artifices, piècetrès nécessaire à tous ceux qui font profession d'éloquence ».Rouen, 1623.

7. À bien lire la bibliographie qui se trouvelà la fin de votre texte del'Encyclopédie de laPléiade (p. 653), le termesuggestifque vous utilisez afin de définir l'essai de Claude Roy :Arts baroques, indique bien les réticences que l'on peut avoir vis-à-vis d'un tel livre.

8. QuatremèreDE QUINCY :Histoire de la vie et des ouvrages des plus célèbres architectesduXI siècle jusqu'à la fin duxviII ${ }^{\mathrm{e}}$ siècle. Paris, Jules Renouard, 1830.

9.

Une étude récente fut menée par Marie-Madeleine Dujon :

À la recherche du regard sur l'œuvre de Delacroix.Paris, Université de Paris I

,1979. Cet excellent travail montrait bien - sur un sujet fort peu baroque - comment s'inscrivaient dans les discours - sur plus d'un siècle et demi - les diverses manières de regarder ou de ne pas regarder l'œuvre tout en parlant. 
10. siècle : Essais d'interprétations, Louis IIde Bavière et ses châteaux.Paris,UniversitéParis I,1977.

11. HEGEL:System, $\$ 454$;Zus.X. 334. Cf. G. LEBRUN :LaPatienceduConcept.Paris. Gallimard,1972,p. 67, hegelienne

note 68. surlaBildung

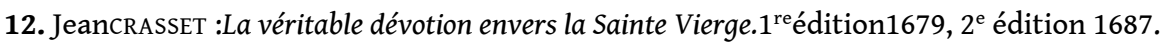

\section{AUTEUR}

\section{JACQUES VANUXEM}

Historien de l'Art 